\title{
The Known Unknowns: Neural Representation of Second-Order Uncertainty, and Ambiguity
}

\author{
Dominik R. Bach, Oliver Hulme, William D. Penny, and Raymond J. Dolan \\ Wellcome Trust Centre for Neuroimaging, University College London, London WC1N 3BG, United Kingdom
}

Predictions provided by action-outcome probabilities entail a degree of (first-order) uncertainty. However, these probabilities themselves can be imprecise and embody second-order uncertainty. Tracking second-order uncertainty is important for optimal decision making and reinforcement learning. Previous functional magnetic resonance imaging investigations of second-order uncertainty in humans have drawn on an economic concept of ambiguity, where action-outcome associations in a gamble are either known (unambiguous) or completely unknown (ambiguous). Here, we relaxed the constraints associated with a purely categorical concept of ambiguity and varied the second-order uncertainty of gambles continuously, quantified as entropy over second-order probabilities. We show that second-order uncertainty influences decisions in a pessimistic way by biasing second-order probabilities, and that second-order uncertainty is negatively correlated with posterior cingulate cortex activity. The category of ambiguous (compared with nonambiguous) gambles also biased choice in a similar direction, but was associated with distinct activation of a posterior parietal cortical area; an activation that we show reflects a different computational mechanism. Our findings indicate that behavioral and neural responses to second-order uncertainty are distinct from those associated with ambiguity and may call for a reappraisal of previous data.

\section{Introduction}

Probabilistic action-outcome associations provide uncertain predictions of the future, even when their probabilities are known precisely, which we refer to as "first-order uncertainty." However, action-outcome probabilities themselves can be endowed with imprecision, that is, "second-order uncertainty." Understanding how uncertainty is encoded is paramount for all probabilistic accounts of brain function (Friston, 2010), and tracking second-order uncertainty in particular is indispensable for optimal decision making (Daw et al., 2005), and associative learning (Pearce and Hall, 1980; Friston, 2010).

Economic theory furnishes a means to experimentally manipulate uncertainty in an explicit manner, where first-order uncertainty is usually conceptualized as "risk" (von Neumann and Morgenstern, 1944), and distinguished from second-order uncertainty (Knight, 1921), referred to as "ambiguity" (Ellsberg, 1961). The distinction arises from observations that when winning probabilities of lotteries are known (nonambiguous) or completely unknown (ambiguous), individuals avoid ambiguous lotteries even when this avoidance is costly (for review, see Camerer, 1999). Furthermore, neural responses to ambiguity in posterior parietal cortex, posterior dorsolateral prefrontal cortex and anterior insula (Huettel et al., 2006; Bach et al., 2009) suggest a

Received March 22, 2010; revised Nov. 1, 2010; accepted Nov. 22, 2010.

This work was funded by a programme grant from the Wellcome Trust and a Max Planck Research Award to R.J.D., and by a personal grant from the Swiss National Science Foundation to D.R.B. We thank Karl Friston for his comments on this manuscript. Rosalyn Moran and Mkael Symmonds contributed to the design of the experiments; and Peter Dayan provided constructive discussion of the results.

Correspondence should be addressed to Dominik R. Bach, Wellcome Trust Centre for Neuroimaging, 12 Queen Square, London WC1N 3BG, UK. E-mail: d.bach@fil.ion.ucl.ac.uk.

DOI:10.1523/JNEUROSCI.1452-10.2011

Copyright $\odot 2011$ the authors $\quad 0270-6474 / 11 / 314811-10 \$ 15.00 / 0$ representation that is distinct from those supporting first-order uncertainty in economic gambles (Dreher et al., 2006; Preuschoff et al., 2006; Tobler et al., 2007).

However, ambiguous and nonambiguous lotteries differ in several aspects other than second-order uncertainty. Therefore, it remains unclear whether ambiguity aversion and neural responses to ambiguity reflect second-order uncertainty. Ambiguity aversion is diminished when choices are made privately instead of publicly (Curley et al., 1986; Trautmann et al., 2008), and when unfavorable first-order probabilities are precluded (Larson, 1980; Keren and Gerritsen, 1999). Both ambiguity aversion (Chow and Sarin, 2002) and neural responses to ambiguity (Bach et al., 2009) are greatest when missing information about first-order probabilities is potentially knowable (i.e., known to the experimenter, and usable to infer the conditional first-order probabilities) (see also O'Neill and Kobayashi, 2009). These findings suggest that factors other than second-order uncertainty may confound experimental manipulations of ambiguity, such as beliefs about the availability of missing knowledge to oneself and to others, e.g., the idea that one is being cheated, or that one has to take action to get at the missing bit of information.

Hence, in this study we sought to quantify second-order uncertainty in ambiguous economic gambles and establish its specific neural correlates. Additionally, by contrasting ambiguous and nonambiguous gambles, we could determine whether neuronal responses differed from those evoked specifically by second-order uncertainty. Any difference would argue that categorical manipulations of ambiguity may not serve as a "pure insertion" of second-order uncertainty (Friston et al., 1996). Experimentally, we depart from standard economic experimental approaches that provide either all or no information about firstorder probabilities, and constructed ambiguous gambles, where 
some information is given in the form of second-order probabilities that specify which conditional first-order probability will be realized. By showing participants two colored balls on the edges of a screen, each denoting a first-order probability of getting electric shocks (Fig. 1), we could vary the amount of this information (the second-order uncertainty) continuously, and quantify it by its Shannon entropy (Shannon, 1948).

\section{Materials and Methods}

Participants. Twenty-four healthy right-handed individuals took part in the behavioral experiment (12 male, 12 female, mean age \pm SD: $25.3 \pm 4.5$ years), and an independent sample of 20 healthy right-handed individuals participated in the imaging experiment ( 12 male, 8 female, mean age \pm SD: $22.9 \pm 2.5$ years). The absence of visual impairments and neurological or psychiatric symptoms/disorders was assessed via questionnaire. For the imaging experiment, handedness was controlled with the Edinburgh Handedness Inventory (Oldfield, 1971) (mean \pm SD: $85.3 \pm 22.1$ ). Participants were recruited from the general population by advertisement and were given a monetary compensation of $\mathfrak{k} 30$ (behavioral experiment) or $\mathfrak{£} 40$ (imaging experiment). All participants gave written informed consent, and the study was approved by a National Health Service research ethics committee.

Design. Our experiments took the following general form. Participants learned that the color of a ball presented on a computer screen was associated with a certain conditional firstorder probability of receiving three aversive electric shocks (Fig. 1A). On any trial, participants could either choose to accept a gamble on this ball, or receive a single electric shock of the same intensity. After learning these first-order probabilities, the gamble was rendered ambiguous by presenting two colored balls at the opposite edges of the screen and a ball silhouette in between (Fig. 1B). Participants were instructed that the two colored balls corresponded to two "bowling ball players," one of which would play his ball onto the lane. The ball silhouette was the final position of the played ball, and was more likely to come from either of the two "players," and thus represent either of the two colored balls, the closer it was to this ball. To solve this problem optimally, one needs to infer the second-order probabilities that either colored ball would be sampled on the basis of how close the silhouette was to them, then combine these with the two conditional first order probabilities, hence compute the expected first-order probability of being shocked, and thus decide whether to accept the gamble or to receive a single electric shock instead.

If the two balls at the edges of the screen have different colors, the second-order probabilities can be inferred when the distributions of the final ball positions are known. These distributions were fixed and indicated by color bars with varying intensity (Fig. $1 B$ ). The second-order uncertainty is then dependent on the silhouette position and was quantified as Shannon entropy of the second-order probabilities:

$$
H=-\sum_{i=1}^{2} p\left(\text { color }_{i} \mid \text { position }\right) * \log \left[p\left(\text { color }_{i} \mid \text { position }\right)\right]
$$
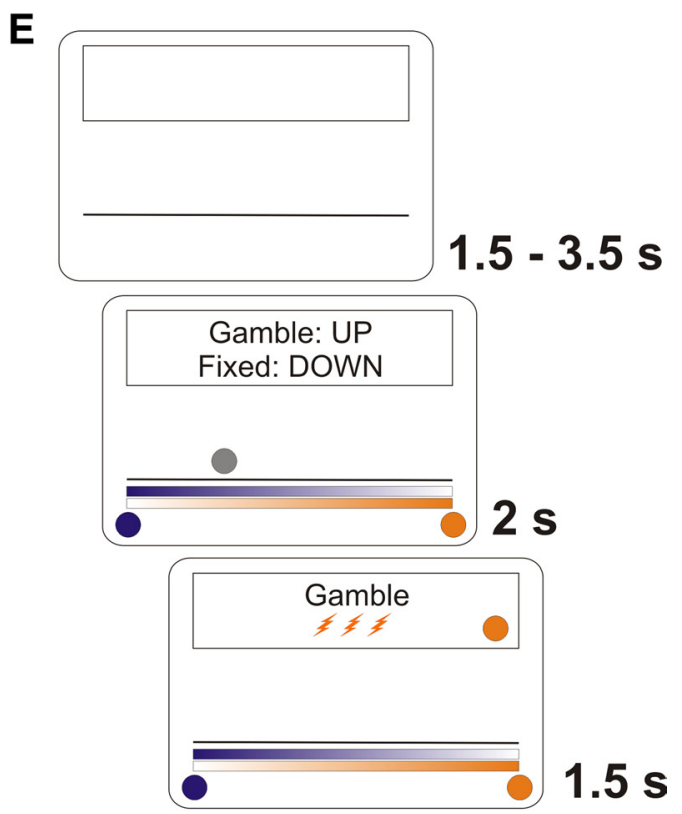

$1.5 \mathrm{~s}$

Figure 1. Experimental setup. $A$, Preceding learning task. In each of 144 trials, participants saw a colored ball. Each of three ball colors represented a gamble (framed as "lottery ticket") with a different first-order probability $(0.2,0.5,0.8)$ of getting three ( ambiguous trials, two colored balls appeared, indicating two possible first-order probabilities. It was explained that these balls represented two bowling ball players, one of which would play his ball that would be shown as a silhouette. Thus, the closer the silhouette appeared to one of the two balls, the more likely it was to represent this ball. Again, a decision had to be made between the gamble and one certain shock. $C$, In 84 additional nonambiguous trials, the two balls had the same color. The position of the robabilities of which conditional first-order probability would be realized, given the silhouette position. It can be seen that this 列 (he

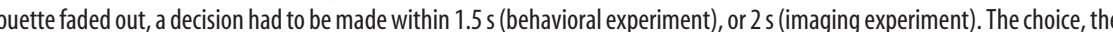
ball that was being played, and the ultimate outcome, were then shown in the feedback box above the bowling ball lane.

where, by Bayes' rule and in the absence of prior knowledge,

$$
p(\text { color } \mid \text { position })=\frac{p(\text { position } \mid \text { color })}{\sum_{i=1}^{2} p\left(\text { position } \mid \text { color }_{i}\right)} .
$$

One can see from Figure $1 D$ that the silhouette is more informative (i.e., has less entropy $H$ ) about the color, the closer it is to one of the colored balls on the edges of the screen. This manipulation incidentally also varies the expected first order probability which was decorrelated from entropy by using six different combinations/orderings of balls, and was additionally taken into account for analysis (see data analysis).

In this way, we were able to measure acceptance of gambles and associated evoked brain responses as a function of the entropy of the secondorder probabilities. This design also allowed us to collapse ambiguous gambles into a factor level to allow a categorical contrast with nonambiguous gambles where both color options were the same (Fig. 1C). In this case, the color of the silhouette can be inferred with certainty, and the prediction is nonambiguous. The expected first order probability is then simply the conditional first order probability associated with the color of both balls. 
Thus, the experiment followed an incomplete factorial design with a discrete factor ambiguity (ambiguous vs nonambiguous) and a continuously varied factor entropy within the ambiguous condition. This design incidentally also varied the additional discrete factors first-order probability $(0.2,0.5,0.8)$ within the nonambiguous and range of first-order probabilities $(0.3,0.6)$ within the ambiguous condition.

Stimuli and gambles. There was one trial for each of 3 ball colors, in combination with the other possible ball colors (ambiguous trials: 2 colors; nonambiguous trials: 1 color), originating position (left or right), and possible ball position (ambiguous trials: 23; nonambiguous trials: 14), resulting in 276 ambiguous and 84 nonambiguous trials, or 360 trials on the whole.

The three ball colors (black, orange, and blue) represented three different lotteries. All three lotteries had the same stake (win: nothing; lose: three electric shocks), but different (first-order) probabilities of being shocked $(0.2,0.5$, and 0.8$)$. The association of color and shock probability was balanced across subjects and learnt in a preceding discrimination learning paradigm involving 144 learning trials (that is, 46 trials per ball color, see Procedure).

Each trial started with a variable interval of 2-4 s (behavioral experiment) or 1.5-3.5 s (imaging experiment), during which a lane and an empty feedback box were visible. Then, two colored balls were shown. Above the balls, two color bars with varying intensity indicated the probability that either ball would land on a given position on the lane if played. This followed a linear probability density function with a maximum at the position of this ball and reaching zero at the position of the opposite ball. A gray silhouette appeared somewhere on the lane $0.5 \mathrm{~s}$ later (behavioral experiment) or immediately (imaging experiment).

The position of the silhouette was determined as follows: the lane was divided into 23 (ambiguous trials) or 14 (nonambiguous trials) intervals of equal cumulative landing probability. The interval in which the ball occurred on a given trial was pseudo-randomized and kept constant across all participants. This ensured that the entropy variation was approximately the same for each participant. We randomly drew the exact position within the landing interval from the corresponding probability density function, and this exact position was taken into account for analysis. The gray silhouette also served as a timer, fading away within $1.5 \mathrm{~s}$ (behavioral experiment) or $2 \mathrm{~s}$ (imaging experiment). Within this time, participants had to make a decision to either accept or reject the lottery by pressing an up or down arrow key on a standard computer keyboard.

The association of up and down button presses and reject or accept was balanced across subjects and kept constant for each participant. It was signaled on each trial (e.g., up arrow: fixed, down arrow: gamble), where the response option associated with the up key was always presented on top of the other option to avoid stimulus-response incongruencies. If participants did not make a choice, they received three electric shocks with certainty, and these trials were excluded from analysis. After the decision time was over, we signaled which color was actually sampled (and thus, the conditional first-order probability) to emphasize that it was predetermined and consistent (Bach et al., 2009). This was shown in a separate feedback box on the top half of the screen, together with an indication of the participant's choice (fixed option or gamble) and an indication of the electric stimulations they would receive (one or three lightning-style electric shock signs, or the words no shock). This feedback was visible for $1.5 \mathrm{~s}$. At the same time, electric stimulations were delivered as a $500 \mathrm{~Hz}$ train of electrical pulses (square wave, individual pulse duration: $1 \mathrm{~ms}$, total duration: $100 \mathrm{~ms}, 400 \mathrm{~V}$, mean current $\pm \mathrm{SD}$, behavioral experiment: $0.076 \pm 0.044 \mathrm{~mA}$, imaging experiment: $0.073 \pm 0.037$ $\mathrm{mA}$ ) via a pin-shaped electrode attached to the left forearm, repeated once (fixed option) or three times with $0.4 \mathrm{~s}$ inter stimulus interval (gamble). Stimulation intensity was the same for the fixed option and gamble, and set in a pre test assessment as slightly below the pain threshold (see Procedure). In 12 of 20 participants in the imaging experiment, 14 random stimuli below the pain threshold were rated for intensity before and after the experiment, and there were no differences in the mean ratings $(p>0.50)$.

Procedure. Upon arrival in the laboratory, the procedure was explained to participants in detail by standardized written and additional oral instructions (see supplemental material, available at www.jneurosci.org).

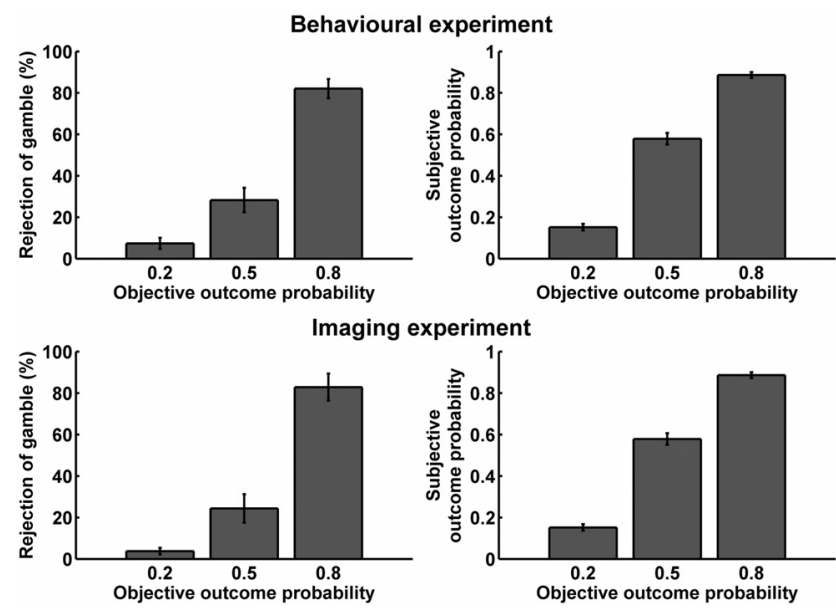

Figure 2. In a preceding learning task consisting of 144 trials, participants learned the outcome probabilities of the three colored balls. Choices for the last 24 balanced trials of this part are shown on the left. After the experiment proper, participants were asked for explicit estimates of the outcome probabilities of the three colored balls, as shown on the right. All results are mean $\pm S E$ for participants that were included into the analysis (behavioral experiment: $n=24$, imaging experiment, $n=20$ ). Participants whose choice proportions and subjective probability estimates did not monotonically vary with outcome probability were excluded from scanning/analysis.

Then, discomfort and pain thresholds for the electric stimulation were assessed with ascending stimulation intensity. Shocks with randomly determined intensity below the pain threshold were then delivered and rated by the participants to establish a stimulus-perception relationship. Stimulus intensity was set slightly below the pain threshold to a clear discomfort level. The same stimulus intensity was used for the single fixed shock and the three shocks delivered after losing a gamble.

In a discrimination learning paradigm closely related to the bowling ball game, participants could explore the three different gambles. One bowling ball was presented at a time, and participants chose to accept or reject the gamble. Otherwise, the procedure was similar to the one described above. Participants played 5 training trials without electric shocks to habituate them to the task. Then, 144 trials followed in two $8 \mathrm{~min}$ blocks. The last 24 trials were fully balanced and served to probe whether participants ordered their preference according to the ordering of objective outcome contingencies (Fig. 2). Nine (behavioral experiment) and 7 (imaging experiment) additional participants who did not have this ordering of preference were not included in the experiment proper. (Note that this procedure excludes both participants who did not learn the outcome contingencies and those who did not have a preference over one or three electric shocks.) The experiment proper was then explained with standardized written and additional oral instructions (see supplemental material, available at www.jneurosci.org), and participants had 5 training trials without electric shocks. In the imaging experiment, participants went into the scanner at this point and, while field maps were taken, engaged in another 24 trials of the learning part to habituate them to the scanner environment. Another 5 training trials for the experiment proper without electric shocks followed. Then, participants engaged in the experiment proper, containing 360 trials in 5 blocks of 8 min or 72 trials each. Breaks between blocks lasted at least $30 \mathrm{~s}$ and could be extended by participants. After the experiment, participants were asked to rate the (first-order) shock probability for each of the three ball colors (How likely was it that shocks would be delivered after the following ball?) on a horizontal visual analog scale from $0 \%$ to $100 \%$ (Fig. 2).

Image acquisition. Images were acquired on a $3 \mathrm{~T}$ whole-body scanner (Trio, Siemens Medical Systems) with a 12 channel head coil for radiofrequency transmission and signal reception. Field maps were acquired with the standard manufacturer's double echo gradient echo field map sequence [echo time (TE), 10.0 and $12.46 \mathrm{~ms}$; repetition time (TR), 1020 ms; matrix size, $64 \times 64$ ], using 64 slices covering the whole head (voxel size, $3 \times 3 \times 3 \mathrm{~mm})$. 
Whole-brain T1-weighted scans were acquired using a modified driven equilibrium Fourier transform (MDEFT) sequence with optimized parameters as described previously (Deichmann et al., 2004). A total of 176 sagittal partitions were acquired with an image matrix of $256 \times 240$ (read $\times$ phase) and twofold oversampling in read direction (head/foot direction) to prevent aliasing (isotropic spatial resolution 1 $\mathrm{mm} ; \alpha, 16^{\circ}$; TR/TE/inversion time, $7.92 \mathrm{~ms} / 2.48 \mathrm{~ms} / 910 \mathrm{~ms}$; bandwidth, $195 \mathrm{~Hz} /$ pixel). Special RF excitation pulses were used to compensate for B1 inhomogeneities of the transmit coil in superior/inferior and anterior/posterior directions. Images were reconstructed by performing a standard three-dimensional (3D) Fourier transform, followed by modulus calculation. No data filtering was applied in $k$-space or in the image domain.

For functional images, we used blood oxygenation level-dependent (BOLD) signal-sensitive $T_{2}{ }^{*}$-weighted transverse single-shot gradientecho echoplanar imaging (EPI) (flip angle $\alpha, 90^{\circ}$; bandwidth, $2298 \mathrm{~Hz} /$ pixel; phase-encoding (PE) direction, anterior-posterior; TE, $30 \mathrm{~ms}$; TR, $3264 \mathrm{~ms}$ ). The manufacturer's standard automatic 3D-shim procedure was performed at the beginning of each experiment. Each volume contained 48 slices of $2 \mathrm{~mm}$ thickness ( $1 \mathrm{~mm}$ gap between slices; field of view, $192 \times 192 \mathrm{~mm}^{2}$; matrix size, $64 \times 64$ ). BOLD sensitivity losses in the orbitofrontal cortex and the amygdala due to susceptibility artifacts were minimized by applying a z-shim gradient moment of $-0.4 \mathrm{mT} / \mathrm{m}^{\star} \mathrm{ms}$, a slice tilt of $-30^{\circ}$ and a positive PE gradient polarity (Weiskopf et al., $2006,2007)$. In each of five scanning sessions, 147 functional whole-brain volumes were acquired. The first 5 volumes, or $16.3 \mathrm{~s}$, of each session were discarded to obtain steady-state longitudinal magnetization. Each session was concluded by 8 volumes, or $26.1 \mathrm{~s}$, of rest without stimuli. During EPI, we recorded respiration with a chest belt and heart rate with a pulse oxymeter on the left little finger.

Image analysis. Echoplanar images were generated online using the scanner manufacturer's reconstruction method. Further processing was done using statistical parametric mapping (SPM8; Wellcome Trust Centre for Neuroimaging, London, UK; www.fil.ion.ucl.ac.uk/spm) on Matlab 7.4 (MathWorks). Images were corrected for geometric distortions caused by susceptibility-induced field inhomogeneities. A combined approach was used which corrects for both static distortions and changes in these distortions due to head motion (Andersson et al., 2001; Hutton et al., 2002). The static distortions were calculated for each subject from a field map that was processed using the FieldMap toolbox as implemented in SPM8. Using these parameters, the echoplanar images were then realigned and unwarped, a procedure that allows the measured static distortions to be included in the estimation of distortion changes associated with head motion. No participant moved $>6 \mathrm{~mm}$ in any direction during scanning. The motion-corrected images were then coregistered to the individual's anatomical MDEFT image using a 12-parameter affine transformation, and normalized to the Montreal Neurological Institute T1 reference brain template (resampled voxel size $2 \times 2 \times 2 \mathrm{~mm}$ ). Normalized images were smoothed with an isotropic $8 \mathrm{~mm}$ full-width at half-maximum Gaussian kernel. The time series in each voxel were highpass filtered at $1 / 128 \mathrm{~Hz}$ to remove low-frequency confounds as is standard in SPM8.

We modeled neuronal responses as a mixture of categorical (ambiguity vs nonambiguity) and parametric effects (linear effect of entropy within ambiguous gambles). The categorical effect involves that of entropy (entropy is always zero in nonambiguous and non-zero in ambiguous gambles) but is confounded by a range of qualitative differences between the two conditions, whereas the parametric effect involves variation of entropy alone. Based on previous findings (Bach et al., 2009) we hypothesized that a categorical contrast of ambiguity vs nonambiguity would be driven by factors unrelated to entropy. Hence, different brain regions should be activated in this categorical contrast to that associated with a continuous variation of entropy. Crucially, because entropy and expected first-order probability are both a function of the second-order probabilities, expected first-order probability was taken into account for all analyses involving entropy, such that none of our results can be explained by an effect of expected first-order probability.

Specifically, we modeled trial onset and trial outcome across all blocks as stick functions convolved with a canonical hemodynamic response function (Friston et al., 1994). Trial onset was modeled separately for both trial types and for correct (i.e., making a choice between accepting and rejecting the gamble) and missing responses ( $2.2 \%$ of all trials, range across participants $0-13 \%)$. Only correct responses were analyzed. Range of first-order probabilities (only for ambiguous trials), expected first-order probability, second-order uncertainty, and choice were modeled as parametric modulators for each of the four trial onset regressors and were serially orthogonalized in this order such that the regressor pertaining to second-order uncertainty only explained variance not already explained by range of first-order probabilities and expected firstorder probability. Trial outcome was modeled as a single stick function, parametrically modulated by the number of shocks received.

To account for serial acquisition of different slices in one volume and differences in the shape of the hemodynamic response function, time and dispersion derivatives for each regressor were included into the model. Regressors of no interest were movement parameters derived from the realignment procedure and regressors for variance caused by the cardiac (Glover et al., 2000) and respiratory cycle (Birn et al., 2006), and a separate constant for each block.

In additional analyses, to account for possible condition differences that might be explained by reaction time differences, these were $z$-transformed for each participant, multiplied with a stick function centered at trial onset, convolved with a hemodynamic response function and, together with time and dispersion derivatives, included in the model as separate regressors of no interest (i.e., not orthogonalized to any other regressor). This regressor will explain all variance in hemodynamic responses that relates to reaction times. If some variance in the other regressors can also be explained by reaction times, SPM will disregard variance that is shared between these regressors and thus discard brain responses that could be explained by reaction times.

Because trial onset and outcome were so close together in time (2s), hemodynamic responses to these events will be correlated (for the canonical responses implemented in SPM, $R^{2}=0.56$ ). That means that we cannot effectively disambiguate whether an effect of trial type was caused by the presence of ambiguity, or by the resolution of ambiguity with the outcome. To do so, one would have had to forego presenting outcomes which was not possible within our study design (see Discussion). Crucially however, within ambiguous trials, one can distinguish responses to second-order uncertainty, and response to the resolution of secondorder uncertainty (i.e., second-order surprise, or self-information), because the latter depends on the actual outcome that varies on a trial-by-trial basis and is therefore only mildly correlated with entropy. Additional analyses therefore separated outcome into ambiguous/nonambiguous trials and modeled entropy of the gray silhouette and surprise of the ball outcome (quantified as selfinformation, i.e., negative log of the probability of that ball outcome, given the second-order probabilities).

Statistical parametric maps were generated from linear contrasts of interest, involving only trials where a response was given (ambiguous $>$ nonambiguous trials, entropy, range of possible first-order probabilities in ambiguous trials, first-order probability in nonambiguous trials) in each participant. A second level random effect analysis (RFX) was then performed using one sample $t$ tests on contrast images obtained in each participant for each comparison of interest $(\mathrm{df}=19)$. We report clusters with a voxel-level threshold of $p<0.001$ (uncorrected) and a clusterlevel threshold of $p<0.05$ (cluster-level corrected across the whole brain for familywise error). In regions of interest for which we had prior hypotheses (posterior inferior frontal gyrus/sulcus, anterior insula, posterior parietal cortex, orbitofrontal and dorsomedial prefrontal cortex, amygdala), results were small volume corrected for familywise error within a sphere of $15 \mathrm{~mm}$ diameter around peak coordinates as reported by Bach et al. (2009), Huettel et al. (2006), and Hsu et al. (2005), and reported at a voxel-level threshold of $p<0.05$. This is indicated in the results section and result tables.

Analysis of choice behavior. Our analysis of choice behavior had three goals: (1) to confirm that participants took the expected first-order probability conveyed by the ball position into account when making a decision (i.e., regardless of the second-order uncertainty also conveyed by the ball position); (2) to determine whether participants accounted for the 
Table 1. Summary of models analyzed to account for the influence of ambiguity and second-order uncertainty on choice (see also Fig. 3)

\begin{tabular}{|c|c|c|c|}
\hline \multicolumn{2}{|c|}{ Models accounting for ambiguity } & \multicolumn{2}{|c|}{ Models accounting for ambiguity and second-order uncertainty ${ }^{a}$} \\
\hline Baseline & $\begin{array}{l}\text { Baseline (ideal observer) model, assuming the reduction of } \\
\text { compound lotteries axiom and taking into account the } \\
\text { silhouette position for the calculation of expected } \\
\text { outcome probability }\end{array}$ & Baseline SOP & $\begin{array}{l}\text { Baseline SOP weighting model without additional } \\
\text { parameters to account for entropy }\end{array}$ \\
\hline Utility weighting & $\begin{array}{l}\text { Utility weighting model, using different utility functions for } \\
\text { ambiguous and nonambiguous decisions }\end{array}$ & Utility weighting & $\begin{array}{l}\text { Utility weighting model, assuming modulation of the } \\
\text { utility function in ambiguous trials }\end{array}$ \\
\hline EU weighting additive & $\begin{array}{l}\text { Expected utility additive weighting model, adding a } \\
\text { constant to the expected utility of ambiguous choices }\end{array}$ & EU weighting additive & $\begin{array}{l}\text { Expected utility additive weighting model, adding a } \\
\text { variable amount to the expected utility of } \\
\text { ambiguous choices }\end{array}$ \\
\hline EU weighting multiplicative & $\begin{array}{l}\text { Expected utility multiplicative weighting model, multiplying } \\
\text { the expected utility of ambiguous choices with a constant }\end{array}$ & EU weighting multiplicative & $\begin{array}{l}\text { Expected utility multiplicative weighting model, } \\
\text { multiplying the expected utility of } \\
\text { ambiguous choices with a variable }\end{array}$ \\
\hline Ep weighting & $\begin{array}{l}\text { Expected probability weighting model, nonlinearly } \\
\text { weighting the expected probabilities in ambiguous } \\
\text { choices by exponentiating them with a constant (Hsu et } \\
\text { al., 2005) }\end{array}$ & Ep weighting & $\begin{array}{l}\text { Expected probability weighting model, exponentiating } \\
\text { expected outcome probability of ambiguous choices } \\
\text { with a variable }\end{array}$ \\
\hline Pessimistic weighting & $\begin{array}{l}\text { Pessimistic weighting model, biasing the second-order } \\
\text { probabilities toward the worse scenario in ambiguous } \\
\text { choices (Ghirardato et al., 2004; Huettel et al., 2006) }\end{array}$ & Pessimistic weighting & $\begin{array}{l}\text { "Pessimistic" weighting model, overestimating/ } \\
\text { underestimating the second-order probability of } \\
\text { the worse scenario }\end{array}$ \\
\hline Minimax & $\begin{array}{l}\text { Minimax model, where choice in ambiguous trials is based on } \\
\text { the worse scenario only }\end{array}$ & Not applicable & \\
\hline SOP & $\begin{array}{l}\text { SOP model, in which the conditional expected outcomes are } \\
\text { nonlinearly weighted before being combined with the } \\
\text { unbiased second-order probabilities into an } \\
\text { expected outcome (Segal, 1987; Klibanoff et al., 2005) }\end{array}$ & Combined SOP & $\begin{array}{l}\text { Combined SOP model, in which the second-order } \\
\text { probabilities are additionally weighted by } \\
\text { entropy }\end{array}$ \\
\hline
\end{tabular}

${ }^{a}$ Based on the second-order probability (SOP) model, additional models were formed that accounted for entropy within ambiguous choices by having an additional free parameter that is modulated by mean-centered entropy. EU, Expected utility; Ep, expected probability.

categorical difference between ambiguity and nonambiguity when making a decision and what computational algorithm they used in so doing; and (3) to ascertain whether, and by which computational mechanism, participants accounted for entropy in their choice. Because the categorical difference between ambiguity and nonambiguity also involves entropy, but encompasses additional factors that are inseparable, we needed to ensure that this categorical difference was already accounted for when analyzing the effect of entropy within ambiguous trials. Therefore, in this step, we used the algorithm that best explained the categorical effect of ambiguity, and complemented this algorithm with additional attributes to account for entropy only within ambiguous trials.

The above aims were implemented in a stepwise Bayesian model comparison. To achieve goal 1, a simple decision algorithm was built, based on expected utility theory (von Neumann and Morgenstern, 1944) that uses the average of both conditional first-order probabilities as expected first-order probability (i.e., does not take the ball position into account), weights all possible outcomes $(0,1$, or 3 electric shocks) by a standard power utility function with one free parameter, and computes a choice probability using a softmax rule with another free parameter. This was then compared against a more plausible baseline algorithm (ideal Bayesian or rational observer) where expected first-order probability is a function of second-order and conditional first-order probabilities (i.e., does take the ball position into account).

In addressing goal 2, note that the baseline model was built on the assumption that compound (i.e., hierarchical) lotteries can be reduced to single-stage bets (von Neumann and Morgenstern, 1944) by calculating an expected first-order probability, which would be incompatible with aversion to ambiguity or to second-order uncertainty (Halevy, 2007). We therefore compared the baseline model to a number of models from the economic literature that drop the compound lotteries axiom, all of which are listed in Table 1, described in mathematical detail in the supplemental material (available at www. jneurosci.org as supplemental material) and reviewed comprehensively by Camerer (1999).

As detailed, the model that best explained the categorical difference between ambiguous and nonambiguous gambles, namely the SOP model (see Table 1 for a description), was then complemented to accommodate for an effect of entropy within ambiguous trials (goal 3). The models took an analogous form as for step 2, with the difference that they multiplied the respective free parameter by mean-centered entropy for each ambiguous gamble (with the exception of the minimax model that cannot usefully be modified by entropy and was therefore dropped; Table 1).

Hence, model comparison was conducted in three steps, comparing (1) the simple with the baseline model, (2) the baseline with ambiguity models, and (3) the best ambiguity model with entropy models. This stepwise model comparison is feasible as all steps refer to independent variance components, and is more robust than simultaneous comparison of all possible model combinations. To make allowance for the expected explanatory power of more complex models in the absence of any true difference, model complexity was penalized using Akaike information criterion (AIC) and the Bayesian information criterion (BIC) which led to similar results unless stated otherwise. Note these approaches are simply two different approximations to the true Bayesian model evidence; though not originally motivated from a Bayesian perspective, model comparisons based on AIC are asymptotically equivalent to those based on Bayes factors (Akaike, 1973; Penny et al., 2004) (see also Burnham and Anderson, 2004, for a Bayesian perspective on AIC). We assumed that different individuals might implement different models and performed a random-effects analysis where the structure of the model is assumed to be a random variable across participants as described previously (Stephan et al., 2009). This analysis computes the proportion of individuals that implement each model and an exceedance probability that states how likely it is that each model is more frequent than any other, in the population from which the sample of subjects were drawn. In the absence of conventions on exceedance probabilities, we defined a probability of $p>0.95$ as decisive, to be comparable to standard model comparison. Also, we used family wise comparison of models as pointed out in the results section (Penny et al., 2010). All models used the objective conditional first-order probability. Additional analyses taking account of the subjective probability as stated in postexperimental ratings (see Procedure) led to similar results. To enhance statistical power, data 
from both experiments was combined for choice analysis. Analysis of the individual experiments lead to very similar results.

\section{Results}

\section{Reaction times}

To control for possible effects of ambiguity on decision difficulty, we analyzed reaction times (RT). Responses in ambiguous trials were considerably slower than in nonambiguous trials (behavioral experiment: $987 \pm 44 \mathrm{~ms}$ vs $850 \pm 50 \mathrm{~ms} ; p<$ 0.001; imaging experiment: $1095 \pm 47 \mathrm{~ms}$ vs $958 \pm 39 \mathrm{~ms} ; p<0.001)$. There was no significant correlation between RT and entropy.

\section{Choice behavior associated with ambiguity and entropy}

First, the baseline (ideal observer) model performed better than the simple model (exceedance probability $p>0.999$ ), showing that participants took the ball position into account to compute expected first-order probability, as instructed. Second, we found that models which take account of a categorical difference between ambiguous and nonambiguous gambles were better at explaining the data than the baseline model (exceedance probability $p>0.999$ ). Figure 3 (left) shows the exceedance probabilities (i.e., probabilities that this model is more frequent in the population than any other) for individual models. The model that most frequently accounted for the difference between ambiguous and nonambiguous lotteries was the SOP model (Table 1; see supplemental material, available at www.jneurosci.org, for all formulae). In this model, conditional expected outcomes are nonlinearly weighted by exponentiating them with a constantfor both ambiguous and nonambiguous trials - and then combined using the unbiased second order probabilities (Segal, 1987; Klibanoff et al., 2005). Hence, for a typical ambiguity-averse individual in an ambiguous trial, the combination of two weighted conditional expected outcomes will be less valuable than weighting the average expected outcome under nonambiguous conditions and so explain a difference between ambiguous and nonambiguous lotteries. In economic theory, this is analogous to a nonlinear utility function explaining individual risk preferences. Thirdly, models which account for entropy within ambiguous gambles explained the data better than a model that did not when analyzing AIC ( $p>0.95)$, while BIC with its heavier penalization of additional parameters preferred the simpler model. As shown in Figure 3 (right), the "pessimistic" weighting model performed best where the amount and direction (optimistic vs pessimistic) of a second-order probability weighting was dependent on the actual amount of entropy (i.e., pessimistic weighting with above-average entropy, and vice versa). This is achieved, in the model, by multiplying the second-order probability of the worst outcome scenario with a variable that is greater than one if entropy is higher than average, and smaller than one if entropy is below average. There was even stronger evidence (i.e., for both AIC and BIC) that models which did not use SOP to account for the effect of entropy were more frequent than the model using SOP ( $p>0.99$ ), implying different mechanisms accounting for the effects of ambiguity and entropy.

Together, the results suggest that both the categorical presence of ambiguity (as opposed to nonambiguity) and the amount of entropy within these ambiguous gambles are taken into account when making a decision. However, there is strong evidence that the mechanism by which the decision is influenced is different for the mere presence of ambiguity and for variations in the associated degree of entropy. The algorithm that best explained choice differences between ambiguous and nonambiguous lotteries leaves the second-order probabilities unbiased, whereas the algorithm accounting for entropy biases appears to bias second-order probabilities.

\section{Brain regions responsive to entropy and ambiguity}

Our modeling data pointed to distinct processes associated with ambiguity and its entropy. Thus, we used fMRI to test the validity of our model in so far as it predicts dissociable brain responses to categorical ambiguity on the one hand and its entropy on the other. Data are summarized in Table 2. The main contrast of interest was the effect of entropy within ambiguous trials. Here, we found an association between entropy and BOLD responses in bilateral posterior midline areas, including the posterior cingulate and cuneus, extending laterally into adjacent parts of the parietal, occipital, and temporal cortices, and also into areas outside of the gray matter, possibly due to between-subjects anatomical variability and smoothing (Fig. 4A-D; estimated BOLD responses in supplemental Fig. S1, available at www.jneurosci.org as supplemental material). These BOLD responses were greater when entropy was lower with no responses observed for the opposite contrast. To exclude that these responses were caused by the surprise of outcomes (which is, on the average, related to entropy), we modeled outcomes separately for the two trial types, and added a regressor accounting for the instantaneous secondorder surprise of the ball outcome in ambiguous trials. This revealed the same association of entropy with BOLD responses in a slightly smaller cluster (4925 voxels). Positive responses to surprise in the posterior parietal cortex failed to reach whole-brain level corrected significance $(p=0.06)$, whereas negative responses to surprise were found in the medial right cuneus (see supplemental Table S1, available at www.jneurosci.org as supplemental material). To exclude the possibility that choice might explain brain activations relating to second-order uncertainty, we switched the serial position of the regressors for second-order uncertainty and choice. This did not impact on our reported results and brain responses were similar in location and extent (4996 voxels).

In contrast, a categorical difference between ambiguous and nonambiguous trials (Table 2, Fig. 4E) (estimated BOLD responses in supplemental Fig. S1, available at www.jneurosci.org as supplemental material) showed enhanced BOLD responses in left posterior parietal cortex (pPAR), overlapping with clusters found previously (Huettel et al., 2006; Bach et al., 2009). To exclude reaction time differences between conditions as explaining 
Table 2. BOLD responses to contrasts of interest

\begin{tabular}{|c|c|c|c|c|c|}
\hline Brain regions & $\begin{array}{l}\text { Brodmann area of } \\
\text { local maxima }\end{array}$ & Hemisphere & Voxel number & Voxel $t$ score & $\begin{array}{l}\text { Montreal Neurological Institute brain template } \\
\text { coordinates of local maxima }\end{array}$ \\
\hline \multicolumn{6}{|l|}{$\begin{array}{l}\text { Responses to low second-order uncertainty (i.e. entropy) } \\
\text { within ambiguous gambles }\end{array}$} \\
\hline $\begin{array}{l}\text { Precuneus, superior and inferior parietal lobule, } \\
\text { postcentral gyrus }\end{array}$ & 7 & Left & 516 & 5.6 & $-16,-54,46 ;-22,-54,52 ;-10,-62,56$ \\
\hline \multicolumn{6}{|l|}{$\begin{array}{l}\text { Ambiguous }>\text { non-ambiguous gambles: correlation with } \\
\text { individual measures of ambiguity aversion }\end{array}$} \\
\hline Inferior frontal gyrus* & 47 & Right & 61 & 5.21 & $44,32,2$ \\
\hline \multicolumn{6}{|l|}{ Low $>$ high probability range within ambiguous gambles } \\
\hline Superior and middle frontal gyrus & 9 and 8 & Left & 154 & 5.78 & $-22,30,36 ;-28,26,40 ;-28,18,48$ \\
\hline Middle frontal gyrus & 9 & Right & 126 & 5.22 & $38,22,42$ \\
\hline
\end{tabular}

All clusters are reported as cluster level corrected with $p<0.05$ for familywise error. Additional clusters from regions of interest are reported at a voxel-level significance threshold of $p<0.001$, small volume corrected with $p<0.05$ for familywise error in regions of interest for a sphere with $15 \mathrm{~mm}$ radius around peak coordinates as reported by Bach et al. (2009) and Huettel et al. (2006) (marked with *). No significant BOLD responses were observed for any other contrast of interest.

${ }^{\dagger}$ Note that in nonambiguous trials, normalized shock probability equals normalized expected value, or utility, as value/utility was not varied throughout the experiment.

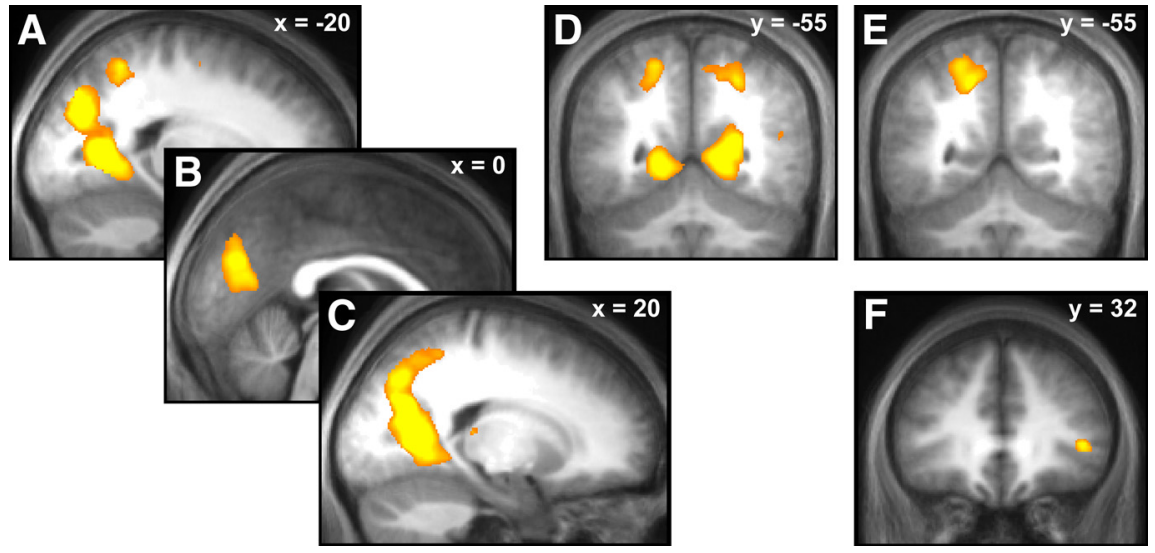

Figure 4. $\quad A-D, B O L D$ responses to low second-order uncertainty (i.e., Shannon entropy of second-order probabilities) within ambiguous trials. $\boldsymbol{E}$, Different responses were found for the contrast ambiguity vs nonambiguity (note that responses in the posterior parietal cortex, $\boldsymbol{D}$ and $\boldsymbol{E}$, are high for ambiguity where entropy is higher than in nonambiguity, but also high for low entropy within ambiguous trials, suggesting that responses to ambiguity are not driven by entropy). $\boldsymbol{F}$, Responses to ambiguity vs nonambiguity in right inferior frontal gyrus covaries with ambiguity aversion on a between-subject level. All contrasts are overlaid on an average normalized $\mathrm{T} 1$ image from the whole sample and are in neurological convention. Responses in $\boldsymbol{A}-\boldsymbol{E}$ survive whole brain correction for familywise error at a cluster-level threshold of $p<0.05$ and a voxel-level threshold of $p<0.001$; responses in $\boldsymbol{F}$ are small volume corrected for familywise error at a voxel-level threshold of $p<0.05$.

a difference in BOLD response, we covaried these out in an additional analysis. This yielded a smaller (335 voxel) cluster in the same location. No brain region showed a greater response in nonambiguous compared with ambiguous trials, in keeping with a previous observation (Bach et al., 2009). Notably, responses in the pPAR overlapped with those seen for the effect of entropy (compare Fig. 4, $D$ and $E$ ). However, the direction of the effect in this region was in diametrical opposition: the BOLD response in this region was negatively correlated with entropy, but positively correlated with ambiguity. Given the opposite sign of the association, one might conclude that responses to ambiguity are not driven by the difference in entropy between these two conditions, but other factors.
For some individuals, the presence of ambiguity is likely to be more relevant than for others. We explored interindividual differences in the brain response to ambiguity (Fig. 4F) and showed that higher rejection rates for ambiguous gambles (hence: ambiguity aversion) were associated with enhanced responses to ambiguous $>$ nonambiguous gambles in the right inferior frontal gyrus. This cluster was not found in the main contrast ambiguous $>$ nonambiguous gambles but was in the vicinity of a cluster reported previously and survived small volume correction within a sphere around this location (Huettel et al., 2006). There was no association of ambiguity aversion with brain responses to entropy, again underlining the distinction between categorical ambiguity and entropy in ambiguity.

Our design incidentally also varied the range of possible outcome probabilities, and the expected risk probability. The difference between ambiguous trials with a high range of possible outcome probabilities (i.e., between 0.2 and 0.8 ) and those with a low range (i.e., $0.2-0.5$ or $0.5-0.8$ ), represented in the contrast low $>$ high outcome probability range, showed a localized BOLD response in the left prefrontal cortex (see Fig. S2C, available at www.jneurosci.org as supplemental material). This cluster survived small volume correction around peak coordinates reported previously (Huettel et al., 2006). Note that our paradigm requires participants to form neural representations of three previously learned shock probabilities; and behavioral analyses indicated this was the case. In keeping with this, brain responses associated with higher risk probability were observed in the right posterior parietal cortex 
and bilateral dorsolateral prefrontal cortex (see Fig. S2 A, B, available at www.jneurosci.org as supplemental material).

\section{Discussion}

Uncertainty about action-outcome associations pervades most real-life decisions and can be conceptualized in a hierarchical probabilistic model where uncertainty of future outcome predictions is termed first-order uncertainty, and uncertainty about the associations themselves second-order uncertainty. To investigate the neural representation of second-order uncertainty, previous studies have relied on the economic concept of ambiguity where second-order uncertainty can be either zero (no ambiguity) or maximal (ambiguity); however this categorical contrast is confounded with qualitative factors. Hence, in the present study we continuously varied the second-order uncertainty of ambiguous lotteries continuously as the entropy of second-order probabilities and contrasted this with nonambiguous lotteries that did not contain second-order uncertainty.

Three main findings emerge. First, decisions on the lotteries appeared to depend on the amount of second-order entropy such that participants were biased toward avoiding a lottery with high second-order entropy, in keeping with a previous study that qualitatively varied second-order uncertainty in ambiguous gambles but fell short of a precise definition (Keren and Gerritsen, 1999). Bayesian comparison suggested that this effect on choice is achieved by biasing the second-order probabilities toward the worst possible outcome scenario when second-order entropy is high (and vice versa when second-order uncertainty is low). Second, BOLD responses to second-order entropy were found in a large posterior midline area encompassing posterior cingulate cortex (PCC) and precuneus, with stronger responses when entropy was low. Thirdly, we replicate previous findings that the mere presence of ambiguity is avoided when compared with nonambiguous lotteries (Becker and Brownson, 1964; Slovic and Tversky, 1974; Yates and Zukowski, 1976; MacCrimmon and Larson, 1979; Curley et al., 1986; Keren and Gerritsen, 1999). Model comparison indicated that this was achieved by nonlinearly weighting the conditional expected outcomes before combining them into an expected outcome, without biasing second order probabilities (Segal, 1987; Klibanoff et al., 2005). BOLD responses to this contrast were seen in posterior parietal cortex (pPAR), replicating two previous studies (Huettel et al., 2006; Bach et al., 2009). Additionally, within posterior prefrontal cortex BOLD responses in this same contrast varied with the amount of ambiguity aversion on a between-subjects level, an area previously implicated in processing ambiguity (Huettel et al., 2006; Bach et al., 2009).

In interpreting these results, we first note that second-order entropy and the contrast ambiguity vs nonambiguity engage different brain areas and appear to evoke different computational processes. The latter contrast has previously been taken to reflect differences in second-order uncertainty (because nonambiguous lotteries have zero second-order uncertainty), but this disregards confounds such as having to publicly reveal uncertainty attitudes (Curley et al., 1986; Trautmann et al., 2008), the possibility of very unfavorable first-order probabilities (Larson, 1980; Keren and Gerritsen, 1999), and search for potentially knowable information (Chow and Sarin, 2002; Bach et al., 2009). Also, the very logic of ambiguous lotteries seems more complicated than of nonambiguous ones and might therefore be aversive. Our findings suggest that the neural representation of ambiguity may not be driven by second-order uncertainty but may reflect these confounding factors, a conclusion that qualifies previous findings
(Huettel et al., 2006; Bach et al., 2009). At the same time, ambiguity aversion might not be driven by second-order uncertainty either, thus calling into question the conceptualization of ambiguity as "missing information" (Camerer, 1995).

By manipulating second-order uncertainty in a continuous and unconfounded manner as second-order entropy, we demonstrate an encoding in a posterior midline network with greater responses when uncertainty is lower. This effect survives controlling for surprise at the time point of the outcome and is therefore driven by the presence of uncertainty rather than its resolution. Several explanations might account for this. First, our behavioral data indicate that lotteries with high second-order uncertainty are devaluated. Previous fMRI studies have found PCC responses representing reward size or subjective value in different paradigms (Kable and Glimcher, 2007, 2010; Ballard and Knutson, 2009; Peters and Büchel, 2009; Smith et al., 2009). Hence, the stronger signal with lower entropy could be interpreted as reflecting higher subjective value of less uncertain lotteries.

However, other areas commonly associated with subjective value (namely striatum and prefrontal/orbitofrontal regions) do not track second-order entropy in the present task. An alternative possibility therefore is that posterior midline areas implicated in the "default mode" network (Raichle et al., 2001; Buckner et al., 2008) are actively suppressed when processing demands (i.e., uncertainty) are high, an observation in keeping with findings that PCC is involved in retrieval of self-relevant memory (Summerfield et al., 2009), and that its activity predicts task errors ( Li et al., 2007). Similar reasoning has been deployed to explain findings of smaller prefrontal deactivations in easy compared with difficult perceptual decisions (Tosoni et al., 2008; Ho et al., 2009).

However, BOLD responses in PCC can also be conceived as directly related to second-order entropy. Greater responses with lower uncertainty would then point to the notion that precision (or amount of information) is encoded. How this encoding is achieved at a neuronal level remains to be determined although we note a proposal that allows for a representation in a large neural population (for review, see Friston, 2009, 2010). For future investigations it might be useful to reframe the decision making model that best explained the data in a (more general) Bayesian framework with "pessimistic" priors that enjoy more weight when the likelihood function has higher uncertainty. Thus, it would be possible to study the effect of pessimistic priors separately from the effect of the likelihood function to disambiguate effects of subjective value and second-order uncertainty.

With respect to the categorical effect of ambiguity we replicated activation in posterior parietal cortex (pPAR) (Huettel et al., 2006; Bach et al., 2009) which we have previously interpreted as reflecting more demanding expected value calculations. Ambiguity might also engage behavioral mechanisms to find out an unknown bit of information, an explanation we previously used to explain responses in anterior insula/posterior prefrontal cortex. No such responses were found in the present study. We suggest that BOLD responses related to outcome search would possibly not occur with the very short anticipation times implemented in the present study ( $2 \mathrm{~s}$ ) as opposed to longer times $(4.5-6 \mathrm{~s})$ used in previous studies. Note, however, that an effect was found in this area for participants with high ambiguity aversion such that differences between this and the previous study may also relate to sample differences in ambiguity aversion. We did not find any responses in brain areas identified in Hsu et al. (2005), in particular amygdala and orbitofrontal cortex (OFC). Although concerned with ambiguity, the latter study collapsed different kinds of situations involving a lack of information dur- 
ing analysis of fMRI data. Thus, in our view there is no convincing empirical evidence that the amygdala or OFC respond to ambiguity.

Model comparison demonstrated that a second order probability (SOP) model was most frequent in explaining the effect of ambiguity on choice, where conditional expected outcomes are nonlinearly weighted before being combined with the unbiased SOP into an expected outcome (Segal, 1987; Klibanoff et al., 2005). A plethora of models accounting for ambiguity has been proposed in the economic literature, mainly based on theoretical/ axiomatic considerations (for review, see Camerer, 1999); to the best of our knowledge we present the first Bayesian model comparison based on choice data.

Several potential limitations of the present study remain to be resolved. First, although there is strong evidence that ambiguity and second-order entropy are accounted for by different computational mechanisms, we acknowledge that the evidence is weaker in determining the precise model by which second-order entropy is taken into account. Thus, it would be desirable to replicate our findings in an experimental setup specifically designed to distinguish between the most likely models from our study. Also, we did not test alternative formulations of second-order uncertainty (e.g., variance). A limitation concerning BOLD responses to ambiguity is that onset and offset of the lottery were close together in time such that it is not possible to disambiguate responses to the presence of ambiguity and its resolution. Note that this does not discount the BOLD effect of second-order uncertainty where responses to uncertainty itself, and to the surprise of the outcome, could be disambiguated.

Tracking second-order uncertainty is crucial for any hierarchical model of perception or learning, such as hierarchical Bayesian (and related predictive coding schemes) (Friston, 2010) and volatility models used to account for context-sensitive learning (Behrens et al., 2007). Indeed, recent findings suggest that the changes in precision during perceptual discrimination are a key determinant of neuronal activity (Hesselmann et al., 2010). We extend such ideas to explicit decisions by showing that secondorder uncertainty in ambiguous lotteries influences choice, possibly by a mechanism that biases second-order probabilities. This second order uncertainty is negatively correlated with BOLD activity in a posterior midline network encompassing the PCC. However, ambiguity as defined within an economic perspective activates different brain regions and appears to exert its effect on choice via a different mechanism. Hence, we argue that behavioral and neural responses to ambiguity might not be driven by second-order uncertainty, calling for a reinterpretation of previous data, and for further research into the mechanisms by which second-order uncertainty acts to guide learning and decision-making.

\section{References}

Akaike H (1973) Information measures and model selection. Bull Int Stat Inst 20: 277-290.

Andersson JL, Hutton C, Ashburner J, Turner R, Friston K (2001) Modeling geometric deformations in EPI time series. Neuroimage 13:903-919.

Bach DR, Seymour B, Dolan RJ (2009) Neural activity associated with the passive prediction of ambiguity and risk for aversive events. J Neurosci 29:1648-1656.

Ballard K, Knutson B (2009) Dissociable neural representations of future reward magnitude and delay during temporal discounting. Neuroimage 45:143-150.

Becker SW, Brownson FO (1964) What price ambiguity? Or the role of ambiguity in decision making. J Polit Econ 72:62-73.

Behrens TE, Woolrich MW, Walton ME, Rushworth MF (2007) Learning the value of information in an uncertain world. Nat Neurosci 10:1214-1221.

Birn RM, Diamond JB, Smith MA, Bandettini PA (2006) Separating respiratory-variation-related fluctuations from neuronal-activity-related fluctuations in fMRI. Neuroimage 31:1536-1548.

Buckner RL, Andrews-Hanna JR, Schacter DL (2008) The brain's default network: anatomy, function, and relevance to disease. Ann N Y Acad Sci 1124:1-38.

Burnham K, Anderson D (2004) Multimodel inference: understanding AIC and BIC in model selection. Sociol Methods Res 33:260-304.

Camerer C (1995) Individual decision making. In: Handbook of experimental economics (Kagel JH, Roth AE, eds), pp 587-704. Princeton, NJ: Princeton UP.

Camerer C (1999) Ambiguity aversion and non-additive probability: Experimental evidence, models and applications. In: Uncertain decisions: bridging theory and experiments (Luini L, ed), pp 53-80. Boston: Kluwer.

Chow CC, Sarin RK (2002) Known, unknown, and unknowable certainties. Theory Decis 52:127-138.

Curley SP, Yates F, Abrams RA (1986) Psychological sources of ambiguity avoidance. Organ Behav Hum Decis Process 38:230-256.

Daw ND, Niv Y, Dayan P (2005) Uncertainty-based competition between prefrontal and dorsolateral striatal systems for behavioral control. Nat Neurosci 8:1704-1711.

Deichmann R, Schwarzbauer C, Turner R (2004) Optimisation of the 3D MDEFT sequence for anatomical brain imaging: technical implications at 1.5 and 3 T. Neuroimage 21:757-767.

Dreher JC, Kohn P, Berman KF (2006) Neural coding of distinct statistical properties of reward information in humans. Cereb Cortex 16:561-573.

Ellsberg D (1961) Risk, ambiguity, and the Savage axioms. Q J Econ 75:643-669.

Friston K (2009) The free-energy principle: a rough guide to the brain? Trends Cogn Sci 13:293-301.

Friston K (2010) The free-energy principle: a unified brain theory? Nat Rev Neurosci 11:127-138.

Friston KJ, Jezzard P, Turner R (1994) Analysis of functional MRI timeseries. Hum Brain Mapp 1:153-171.

Friston KJ, Price CJ, Fletcher P, Moore C, Frackowiak RS, Dolan RJ (1996) The trouble with cognitive subtraction. Neuroimage 4:97-104.

Ghirardato P, Maccheroni F, Marinacci M (2004) Differentiating ambiguity and ambiguity attitude. J Econ Theory 118:133-173.

Glover GH, Li TQ, Ress D (2000) Image-based method for retrospective correction of physiological motion effects in fMRI: RETROICOR. Magn Reson Med 44:162-167.

Halevy Y (2007) Ellsberg revisited: an experimental study. Econometrica 75:503-536.

Hesselmann G, Sadaghiani S, Friston KJ, Kleinschmidt A (2010) Predictive coding or evidence accumulation? False inference and neuronal fluctuations. PLoS One 5:e9926.

Ho TC, Brown S, Serences JT (2009) Domain general mechanisms of perceptual decision making in human cortex. J Neurosci 29:8675-8687.

Hsu M, Bhatt M, Adolphs R, Tranel D, Camerer CF (2005) Neural systems responding to degrees of uncertainty in human decision-making. Science 310:1680-1683.

Huettel SA, Stowe CJ, Gordon EM, Warner BT, Platt ML (2006) Neural signatures of economic preferences for risk and ambiguity. Neuron 49:765-775

Hutton C, Bork A, Josephs O, Deichmann R, Ashburner J, Turner R (2002) Image distortion correction in fMRI: A quantitative evaluation. Neuroimage 16:217-240.

Kable JW, Glimcher PW (2007) The neural correlates of subjective value during intertemporal choice. Nat Neurosci 10:1625-1633.

Kable JW, Glimcher PW (2010) An "as soon as possible" effect in human intertemporal decision making: behavioral evidence and neural mechanisms. J Neurophysiol 103:2513-2531.

Keren G, Gerritsen LEM (1999) On the robustness and possible accounts of ambiguity aversion. Acta Psychol (Amst) 103:149-172.

Klibanoff P, Marinacci M, Mukerji S (2005) A smooth model of decision making under ambiguity. Econometrica 73:1849-1892.

Knight FH (1921) Risk, uncertainty and profit. Boston: Houghton Mifflin.

Larson JR (1980) Exploring the external validity of a subjectively weighted utility model of decision making. Organ Behav Hum Perform 26:293-304. 
Li CS, Yan P, Bergquist KL, Sinha R (2007) Greater activation of the "default" brain regions predicts stop signal errors. Neuroimage 38:640-648.

MacCrimmon KR, Larson S (1979) Utility theory: axioms versus 'Paradoxes.' In: Expected utility hypotheses and the Allais Paradox (Allais M, Hagen O, eds), pp 333-410. Dordrecht: D. Reidel.

Oldfield RC (1971) The assessment and analysis of handedness: the Edinburgh inventory. Neuropsychologia 9:97-113.

O’Neill M, Kobayashi S (2009) Risky business: disambiguating ambiguityrelated responses in the brain. J Neurophysiol 102:645-647.

Pearce JM, Hall G (1980) A model for pavlovian learning — variations in the effectiveness of conditioned but not of unconditioned stimuli. Psychol Rev 87:532-552.

Penny WD, Stephan KE, Mechelli A, Friston KJ (2004) Comparing dynamic causal models. Neuroimage 22:1157-1172.

Penny WD, Stephan KE, Daunizeau J, Rosa MJ, Friston KJ, Schofield TM, Leff AP (2010) Comparing families of dynamic causal models. PLoS Comput Biol 6: e1000709.

Peters J, Büchel C (2009) Overlapping and distinct neural systems code for subjective value during intertemporal and risky decision making. J Neurosci 29:15727-15734.

Preuschoff K, Bossaerts P, Quartz SR (2006) Neural differentiation of expected reward and risk in human subcortical structures. Neuron 51:381-390.

Raichle ME, MacLeod AM, Snyder AZ, Powers WJ, Gusnard DA, Shulman GL (2001) A default mode of brain function. Proc Natl Acad Sci U S A 98:676-682.

Segal U (1987) The Ellsberg paradox and risk-aversion-an anticipated utility approach. Int Econ Rev 28:175-202.

Shannon CE (1948) A mathematical theory of communication. Bell Syst Tech J 27: 379-423:623-656.
Slovic P, Tversky A (1974) Who accepts Savage's axiom? Behav Sci 19:368-373.

Smith BW, Mitchell DG, Hardin MG, Jazbec S, Fridberg D, Blair RJ, Ernst M (2009) Neural substrates of reward magnitude, probability, and risk during a wheel of fortune decision-making task. Neuroimage 44:600-609.

Stephan KE, Penny WD, Daunizeau J, Moran RJ, Friston KJ (2009) Bayesian model selection for group studies. Neuroimage 46:1004-1017.

Summerfield JJ, Hassabis D, Maguire EA (2009) Cortical midline involvement in autobiographical memory. Neuroimage 44:1188-1200.

Tobler PN, O'Doherty JP, Dolan RJ, Schultz W (2007) Reward value coding distinct from risk attitude-related uncertainty coding in human reward systems. J Neurophysiol 97:1621-1632.

Tosoni A, Galati G, Romani GL, Corbetta M (2008) Sensory-motor mechanisms in human parietal cortex underlie arbitrary visual decisions. Nat Neurosci 11:1446-1453.

Trautmann ST, Vieider FM, Wakker PP (2008) Causes of ambiguity aversion: known versus unknown preferences. J Risk Uncertainty 36:225-243.

von Neumann J, Morgenstern O (1944) Theory of games and economic behavior. Princeton, NJ: Princeton UP.

Weiskopf N, Hutton C, Josephs O, Deichmann R (2006) Optimal EPI parameters for reduction of susceptibility-induced BOLD sensitivity losses: a whole brain analysis at $3 \mathrm{~T}$ and $1.5 \mathrm{~T}$. Neuroimage 33:493-504.

Weiskopf N, Hutton C, Josephs O, Turner R, Deichmann R (2007) Optimized EPI for fMRI studies of the orbitofrontal cortex: compensation of susceptibility-induced gradients in the readout direction. Magn Reson Materials Phys Biol Med 20:39-49.

Yates JF, Zukowski LG (1976) Characterization of ambiguity in decisionmaking. Behav Sci 21:19-25. 